\title{
Multifunctionality and control of the crumpling and unfolding of large-area graphene
}

\author{
Jianfeng Zang', Seunghwa Ryu ${ }^{2}$, Nicola Pugno ${ }^{3}$, Qiming Wang ${ }^{1}$, Qing Tu', Markus J. Buehler ${ }^{2}$ \\ and Xuanhe Zhao ${ }^{1 \star}$
}

Crumpled graphene films are widely used, for instance in electronics ${ }^{1}$, energy storage ${ }^{2,3}$, composites $^{4,5}$ and biomedicine ${ }^{6}$. Although it is known that the degree of crumpling affects graphene's properties and the performance of graphene-based devices and materials $s^{3,5,7}$, the controlled folding and unfolding of crumpled graphene films has not been demonstrated. Here we report an approach to reversibly control the crumpling and unfolding of large-area graphene sheets. We show with experiments, atomistic simulations and theory that, by harnessing the mechanical instabilities of graphene adhered on a biaxially pre-stretched polymer substrate and by controlling the relaxation of the pre-strains in a particular order, graphene films can be crumpled into tailored self-organized hierarchical structures that mimic superhydrophobic leaves. The approach enables us to fabricate large-area conductive coatings and electrodes showing superhydrophobicity, high transparency, and tunable wettability and transmittance. We also demonstrate that crumpled graphene-polymer laminates can be used as artificial-muscle actuators.

Graphene possesses a unique combination ${ }^{8}$ of extraordinary mechanical, electrical, thermal and optical properties and high specific surface area. The recent capability of synthesizing largescale graphene $\mathrm{e}^{9,10}$ has motivated intensive efforts to integrate the merits of graphene into high-performance devices and materials ${ }^{1-6}$. In these studies and applications, graphene films are generally wrinkled or rippled with smooth undulations $s^{9,11,12}$ and/or crumpled with sharp ridges, folds and vertices ${ }^{1-6,13}$. As deformation of graphene can strongly affect its properties and the performance of graphene-based devices and materials ${ }^{3,5,7,14,15}$, it is highly desirable to control reversible wrinkling and crumpling of graphene. Although it has been shown that thermal expansion and substrate regulation can induce reversible wrinkling of graphene $e^{9,11,16,17}$ and capillary compression can crumple microscopic graphene flakes into particles ${ }^{3,6}$, it is still not clear how to reversibly crumple and unfold large-area graphene films in a controlled manner. Such a capability, however, can potentially advance the performance of graphene-based devices and materials ${ }^{3,5,7}$, as well as open avenues to exploit the unprecedented properties of graphene. Here, we report a simple method to control reversible crumpling and unfolding of large-area graphene films, which yields novel conductive coatings and electrodes that are superhydrophobic, transparent, and feature tunable wettability and transmittance.

A film of few-layer graphene (3-10 layers) is grown on a nickel film by chemical vapour deposition and then transferred to a polydimethylsiloxane (PDMS) stamp and characterized by
Raman microscopy (Supplementary Figs S1 and S2; ref 9). An elastomer film based on acrylic is biaxially stretched to three to five times its original dimensions (that is, pre-strained by $200-400 \%$ ) and held at the pre-stretched state. The graphene film is then transferred to the pre-stretched elastomer substrate by stamping ${ }^{9}$. Thereafter, the pre-strains in the substrate are relaxed sequentially along two pre-stretched directions, as illustrated in Fig. 1a. During relaxation, the lateral dimensions of the transferred graphene film reduce macroscopically by the same ratio as those of the substrate. Microscopically, however, the graphene film develops wrinkles (Fig. 1b) and delaminated buckles (Fig. 1c) when the substrate is relaxed uniaxially, and becomes crumpled (Fig. 1d) when the substrate is relaxed biaxially. If the relaxed substrate is biaxially stretched back, the crumpled graphene film can be unfolded to a relatively flat state (Fig. 1e). The crumpling-unfolding process is reversible over multiple cycles under the control of substrate deformation (Supplementary Fig. S3). The method is also applicable to few-layer graphene grown on copper films (Supplementary Fig. S4).

Now we discuss the underlying mechanisms that control the crumpling and unfolding of graphene through a joint experimental-theoretical-computational analysis. We first focus on the formation of wrinkles and delaminated buckles in graphene under uniaxial compression. As the pre-stretched substrate is gradually relaxed along one direction, the apparent length of the graphene film reduces from $L_{0}$ at the initial (flat) state to $L$ at the present state (Fig. 1a). We define the macroscopic compressive strain in the graphene film along the relaxed direction as $\varepsilon_{\mathrm{G}}=$ $\left(L_{0}-L\right) / L_{0}$. The compressive strain in graphene can be calculated as $\varepsilon_{\mathrm{G}}=\left(\varepsilon_{\text {pre }}-\varepsilon\right) /\left(\varepsilon_{\text {pre }}+1\right)$, where $\varepsilon_{\text {pre }}$ is the pre-strain of the substrate and $\varepsilon$ is the tensile strain in the substrate at the present state. When the compressive strain in the graphene film reaches a critical value, wrinkles develop with an initial wavelength ${ }^{11,18,19}$

$$
\lambda_{0}=2 \pi h\left[\frac{E}{12 \Lambda \mu_{\mathrm{s}}\left(1-v^{2}\right)}\right]^{1 / 3}
$$

where $E$ and $v$ are Young's modulus and Poisson's ratio of graphene, respectively, $\mu_{\mathrm{s}}$ the shear modulus of the substrate taken to be a neo-Hookean material, $h$ the thickness of the graphene film and $\Lambda=\left(1+\left(1+\varepsilon_{\text {pre }}\right)^{3}\right) / 2\left(1+\varepsilon_{\text {pre }}\right)$. Taking $E=1 \mathrm{TPa}, v=0.165$, $\varepsilon_{\text {pre }}=200 \%$ and $\mu_{\mathrm{s}} \approx 20 \mathrm{kPa}$, we obtain $\lambda_{0} \approx 611 \mathrm{~h}$ (ref. 20). Because the number of graphene layers ranges from 3 to 10 , the initial wavelength is evaluated to be $0.6-2.1 \mu \mathrm{m}$, consistent with our experimental results (Fig. 1b; refs 11,19).

\footnotetext{
${ }^{1}$ Soft Active Materials Laboratory, Department of Mechanical Engineering and Materials Science, Duke University, Durham, North Carolina 27708, USA,

${ }^{2}$ Laboratory for Atomistic and Molecular Mechanics, Department of Civil and Environmental Engineering, Massachusetts Institute of Technology,

Cambridge, Massachusetts 02139, USA, ${ }^{3}$ Department of Civil, Environmental and Mechanical Engineering, Università di Trento, via Mesiano, 77 I-38123

Trento, Italy. *e-mail:xz69@duke.edu.
} 

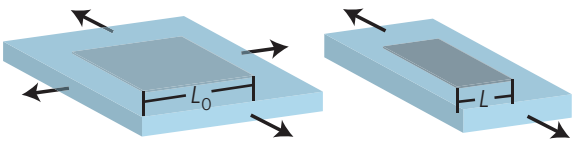

b
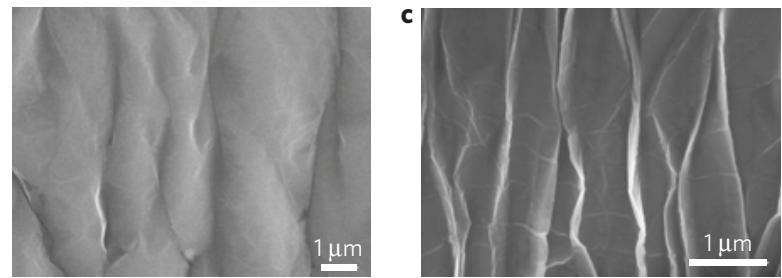

d
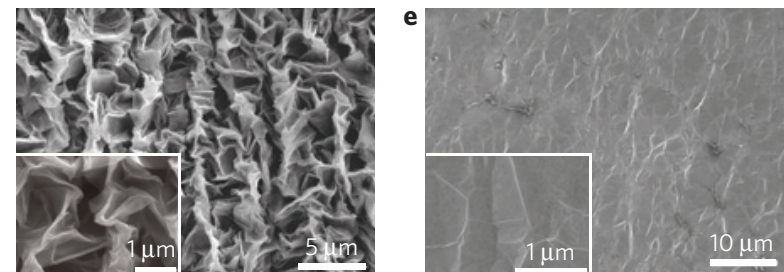

$\mathbf{f}$
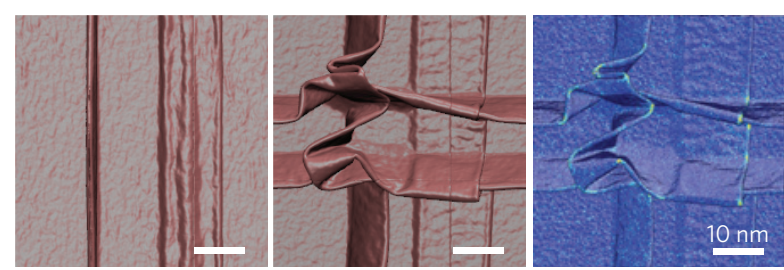

Figure 1 | Controlled crumpling and unfolding of large-area graphene sheets. a, Schematic illustration of macroscopic deformation of a graphene sheet on a biaxially pre-stretched substrate. b-e, SEM images of patterns developed on the graphene sheet: first wrinkles form (b), then delaminated buckles as the substrate is uniaxially relaxed (c), followed by crumples as the substrate is biaxially relaxed (d), which unfold as the substrate is biaxially stretched back (e).f, Atomistic modelling results of the crumpling of a single-layer graphene under uniaxial compression, and biaxial compression, followed by a visualization of the Mises stress distribution (from left to right). Stress concentrations (visualized in red) are observed near highly deformed regions.

Under further uniaxial compression, a pattern of parallel ridges develops with wavelengths of $0.2-2 \mu \mathrm{m}$ (Fig. 1c and Supplementary Fig. S5a). By sectioning the graphene film (Supplementary Fig. S6), we find that the ridges are due to buckling of delaminated regions of the graphene on substrate. The delaminated buckles may initiate from the hills of the wrinkles of graphene ${ }^{21}$ and/or defects on the graphene/polymer interface ${ }^{21-23}$. Once initiated, the delaminated buckles will propagate until the decrease of the graphene-substrate system's elastic energy balances the increase of its interfacial energy $^{21-23}$. Macroscopic and microscopic delaminations of films on compressed substrates have been extensively studied ${ }^{21-23}$ and applied $^{24,25}$. However, to our knowledge, the present study presents the first observation of patterns of delaminated buckles in large-area graphene films on polymer substrates, which is assessed using a close integration of experiment and atomistic simulation.

The crumpling of graphene films under biaxial compression leads to a surface structure that is distinct from the one formed under uniaxial compression. As discussed above, a pattern of parallel delaminated buckles forms in graphene on the substrate when relaxed in one direction (Fig. 1c and Supplementary Fig. S5a). As the substrate is subsequently relaxed in the other direction, the delaminated buckles are compressed along their ridges, and thus buckle and collapse (Fig. 1d and Supplementary Fig. S5b). Furthermore, a new set of delaminated buckles develop perpendicular to the previous ones. The intersection of two orthogonal buckles gives rise to an interesting crumpling pattern with ridges and vertices (Fig. 1d and Supplementary Fig. S5b). Our complementary atomistic simulation reveals high stress concentrations around the ridges and vertices, as shown in Fig. 1f. (Note that the feature size of the patterns from simulation is smaller than that experimentally observed because the simulation considers a single-layer graphene on a rigid surface whereas the experiments are carried out with 3-10 layers of graphene on an elastomer surface.) If the substrate is simultaneously relaxed in the two directions, the crumpling also occurs but leads to more irregular patterns (Supplementary Figs S7b and S8). The difference in crumpling patterns generated by sequential versus simultaneous relaxations is also demonstrated by atomistic simulation (Supplementary Fig. S7 and, Movies S1 and S2). Furthermore, it is noted that the crumpling of delaminated graphene is distinct from the hierarchical folding of perfectly bonded films under biaxial compression that was recently reported $^{26}$. Once the relaxed substrate is biaxially stretched (to its initial length), the parts of the graphene film adhered on the substrate will pull on the delaminated parts, unfolding the crumpled graphene film (Fig. 1e and Supplementary Figs S3 and S5c). If the stretched substrate is relaxed again, the crumpling will reoccur. The graphene film can maintain its integrity over multiple crumplingunfolding cycles (that is, >50) with a few unconnected cracks emerging (Fig. 1e and Supplementary Fig. S3).

The controlled crumpling of graphene leads to self-organized surface structures with controllable feature sizes ranging from nanometres to micrometres (Fig. 1d and Supplementary Fig. S5b), and the hierarchical structure of crumpled graphene can be used for water-repellent and self-cleaning surfaces that mimic the structure of the lotus leaf, for example $e^{27}$. To demonstrate this effect we prepare a crumpled graphene film on a substrate with a biaxial pre-strain of $400 \%$. As shown on Fig. 2a, a water drop placed on top of the crumpled graphene gives a static contact angle of $152^{\circ}$. When the relaxed substrate is biaxially stretched back, the contact angle of the water drop is maintained above $150^{\circ}$ until the biaxial tensile strain in the substrate exceeds $25 \%$ (Fig. $2 \mathrm{~d}$ ). If the substrate is further stretched, the contact angle of the water drop decreases as the crumpled graphene is unfolded (Fig. 2d and Supplementary Fig. S3). Once the graphene is fully unfolded, the contact angle of the water drop decreases to $103^{\circ}$ (Fig. 2b), approximately the same as that of a water drop on a bare substrate (Fig. 2c) owing to the wetting transparency of graphene ${ }^{28}$. Therefore, one can instantaneously tune the wettability of large-area surfaces simply by stretching substrates coated with crumpled graphene, which does not require a complicated fabrication approach ${ }^{29}$.

The tunable wettability of crumpled graphene can also be achieved by stretching substrates with different levels of biaxial prestrains (namely, 250 and 100\% in Fig. 2d). If the water contact angle is re-plotted as a function of the compressive strain in graphene, the curves for different pre-strains collapse onto a universal curve (Fig. 2e). We use the Wenzel and Cassie-Baxter models to explain the water contact angle on crumpled graphene. When the graphene is flat or slightly crumpled, the water will be in conformal contact with the graphene on substrate (that is, the Wenzel state in Fig. 2e). Also, the water will feel the wettability of the polymer substrate owing to the wetting transparency of graphene ${ }^{28}$. On the other hand, if the graphene is highly crumpled, the water drop will sit on a composite of graphene and air (that is, the Cassie-Baxter state in Fig. 2e) and the graphene-air composite is no longer transparent to wetting. Therefore, the apparent contact angle $\theta$ of the water drop can be calculated as

$$
\begin{gathered}
\cos \theta=r \cos \theta_{0}^{\mathrm{S}} \quad(\text { at Wenzel state }) \\
\cos \theta=r\left(1-f_{\mathrm{a}}\right) \cos \theta_{0}^{\mathrm{G}}-f_{\mathrm{a}} \quad(\text { at Cassie-Baxter state })
\end{gathered}
$$




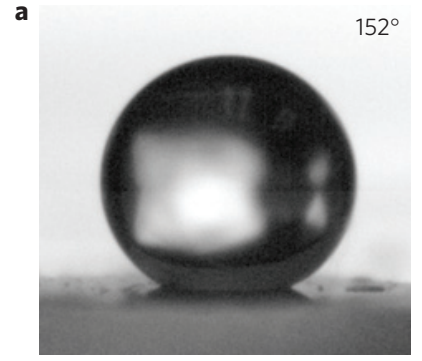

Crumpled graphene

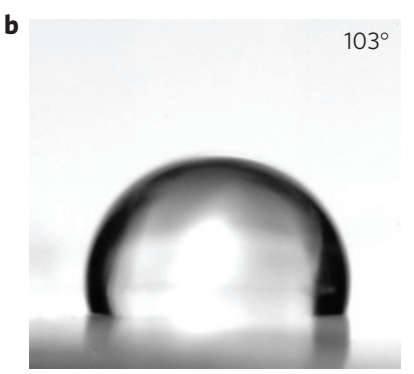

Unfolded graphene

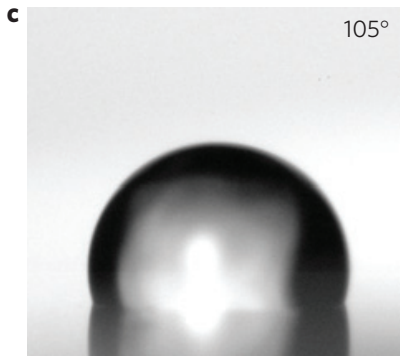

Bare substrate d

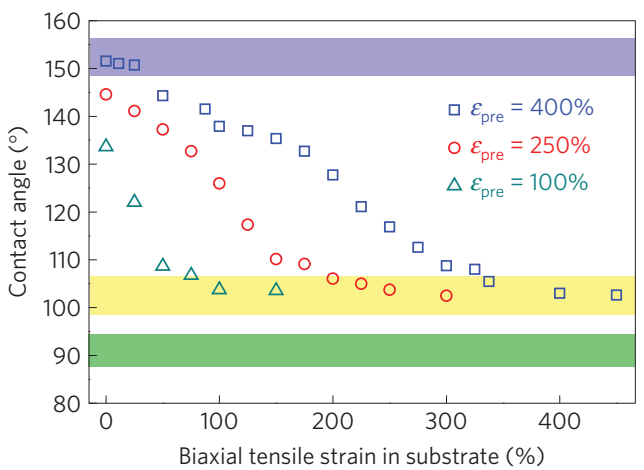

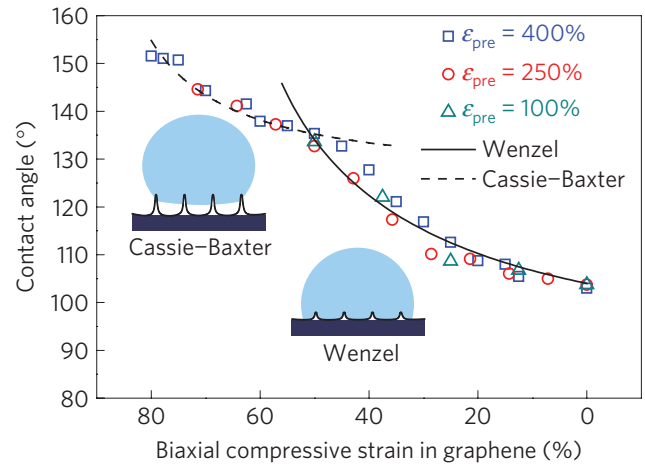

Figure $\mathbf{2}$ | Stretchable graphene coatings capable of superhydrophobicity and tunable wettability. a-c, Images showing the contact angle of a water drop: $152^{\circ}$ on highly crumpled graphene (a), $103^{\circ}$ on unfolded graphene (b) and $105^{\circ}$ on bare substrate (c). d, Contact angle as a function of the biaxial tensile strain in the substrate, $\varepsilon$, with various levels of pre-strain. The contact angle of a water drop on unfolded graphene is closer to that on a bare substrate (yellow band) than that on graphite (green band). e, Contact angle as a function of biaxial compressive strain in graphene, $\varepsilon_{G}$. The experimental results can be explained by our theoretical model. Values in $\mathbf{d}$,e represent the mean of $n$ tests $(n=3-5)$.

where $\theta_{0}^{\mathrm{S}}=105^{\circ}$ and $\theta_{0}^{\mathrm{G}}=90.6^{\circ}$ are the water contact angles on the polymer substrate and graphite respectively, $f_{\mathrm{a}}$ the air fraction in the contact area in the Cassie-Baxter state, and $r$ the roughness of the wetted surface area. The roughness can be calculated by $r=1 /\left(1+d \varepsilon_{\mathrm{G}}\right)^{2}$, where $0<d \leq 1$ takes into account the observed delamination, giving the proportion of compressive strain in graphene that contributes to the roughness. With $d=0.82$ and $f_{\mathrm{a}}=0.61$, our model matches the experimental data very well (Fig. 2e).

The crumpled graphene films can also be used as extremely stretchable and transparent electrodes. To enhance the transparency of crumpled graphene, we pre-stretch the substrate in two directions by unequal pre-strains of 10 and 500\%. Thereafter, the relaxed substrate is uniaxially stretched along the direction with higher pre-strain, while the resistance of the graphene film is measured. The crumpled graphene electrode can maintain good conductivity when the substrate is repeatedly stretched to an extremely high strain of $450 \%$ or highly twisted to an angle of $360^{\circ}$ (Fig. 3a,b). On the other hand, under the same deformations (that is, stretching or twisting), a crumpled gold film of $20 \mathrm{~nm}$ thick develops long and connected cracks with its resistance irreversibly increased by orders of magnitude (Supplementary Fig. S9). The graphene film only begins to fracture significantly when the tensile strain of the substrate exceeds its pre-strain (Supplementary Fig. S10). These results support the notion that a graphene film can maintain its integrity over multiple crumpling-unfolding cycles owing to its high toughness and deformability ${ }^{20}$. Furthermore, when the substrate is stretched, the transmittance of the electrode in the visible range increases from 30 to $80 \%$ as the crumpled graphene is being unfolded (Fig. 3c). The contact angle of a water drop on the graphene electrode can also be varied from $135^{\circ}$ to $103^{\circ}$, as shown in Fig. $3 d$, by stretching the substrate. (Note that our contact-angle model is still valid here, considering $r=1 /\left[\left(1+d \varepsilon_{\mathrm{G} 1}\right)\left(1+d \varepsilon_{\mathrm{G} 2}\right)\right]$, where $\varepsilon_{\mathrm{G} 1}$ and $\varepsilon_{\mathrm{G} 2}$ are compressive strains in graphene along two directions.) The water-repellent capability of the crumpled-graphene electrode can be further enhanced by increasing the biaxial pre-strains of the substrate (for example Fig. 2a). To our knowledge, this combination of stretchability, transparency, and tunability has not been achieved by existing graphene electrodes ${ }^{9}$ or other flexible electrodes based on metal films, conductive polymers, indium tin oxide, nanowires or carbon nanotubes. These properties make crumpled graphene electrodes particularly suitable for niche applications such as actuators and energy harvesters ${ }^{30}$.

Here we demonstrate the application of a laminate of crumpled graphene and dielectric elastomer as a novel artificial-muscle actuator $^{30}$. We biaxially pre-stretch a dielectric-elastomer film by equal pre-strains of $450 \%$, transfer graphene films on its top and bottom surfaces, and then relax the elastomer film to a lower biaxial tensile strain of $300 \%$. As a direct-current voltage of $3,000 \mathrm{~V}$ is applied between the graphene films, the elastomer develops an electric field that induces the Maxwell stress $^{30}$. The Maxwell stress deforms the laminate by reducing its thickness and increasing its area over 100\% (Supplementary Movie S3 and Fig. 4a). The actuation is fast and the grapheneelastomer laminate restores its undeformed state once the voltage is withdrawn. The transmittance of the laminate varies between 40 and $60 \%$ during actuation (Fig. 4b), yielding an artificial muscle with tunable transparency. It is noted that the partial delamination of the graphene film from the substrate is critical to the function of the graphene electrode, where the delaminated part of the graphene enables its high stretchability while the attached part ensures its macroscopically conformal deformation with the elastomer. In contrast, a graphene-elastomer laminate with flat non-delaminated graphene electrodes can only achieve an area strain of $20 \%$ in the first actuation and $7.6 \%$ in the second actuation owing to fracture of the flat graphene electrodes (Supplementary Fig. S11; ref. 9). 
a
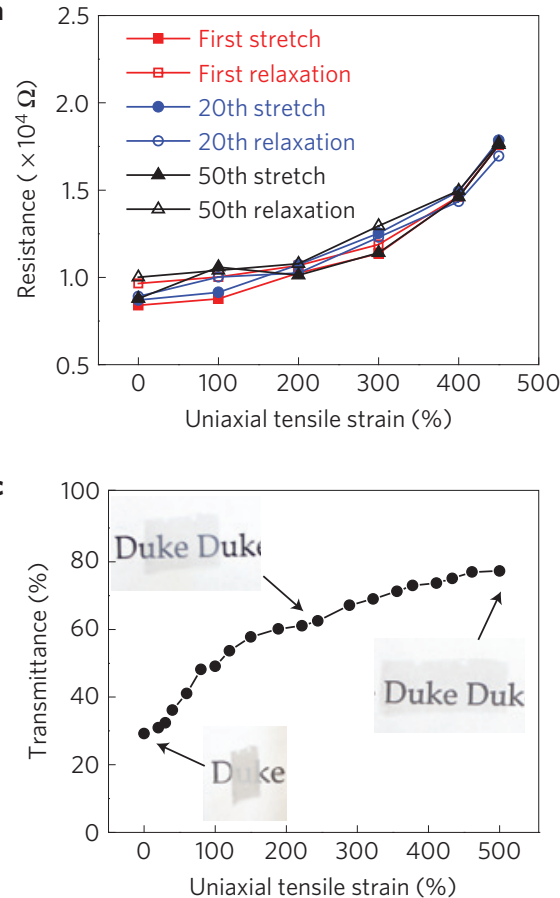

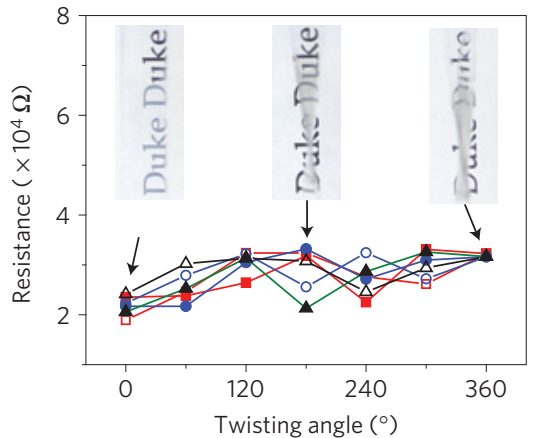

d

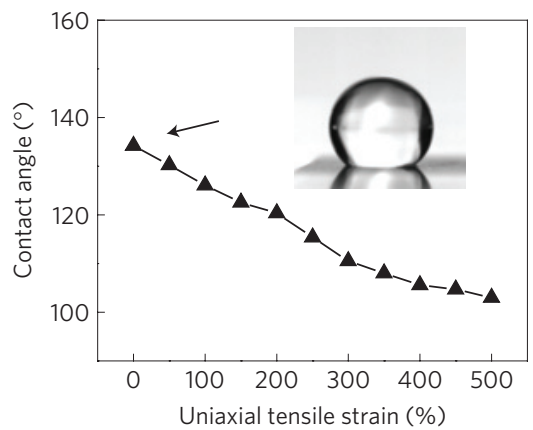

Figure 3 | Graphene electrodes capable of giant stretchability and tunable transparency and wettability. $\mathbf{a}, \mathbf{b}$, Resistance of the electrode on a substrate under multiple cycles of uniaxial tensile strain up to $450 \%$ (a) and twisting up to $360^{\circ}$ (b). The inset shows the electrode under twisting. c, Transmittance of the electrode in the visible range as a function of uniaxial strain in the substrate. The inset shows the electrode under tension. $\mathbf{d}$, Contact angle of a water drop on the electrode as a function of uniaxial strain in the substrate. The inset shows a water drop on the electrode on an undeformed substrate. Values represent the mean of $n$ tests $(n=3-5)$.
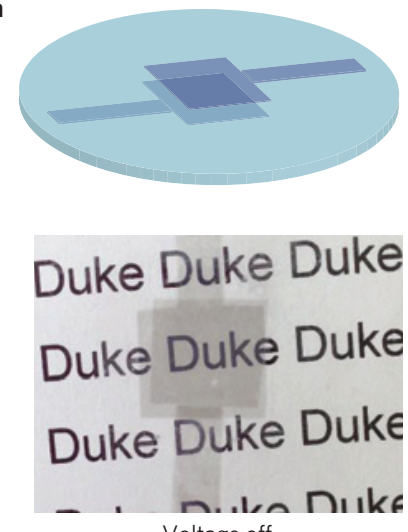

Voltage off

b

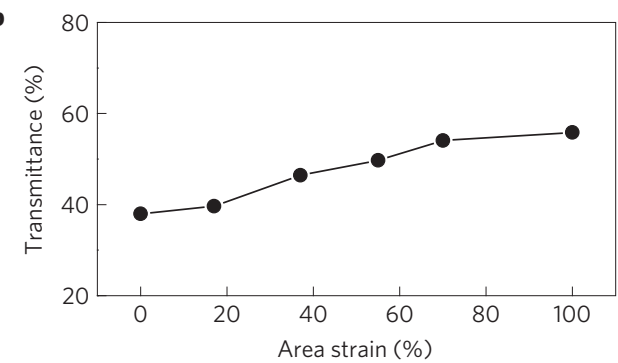

Figure 4 | Voltage-induced actuation of a crumpled graphene-elastomer laminate. a, As a voltage is applied, the laminate reduces its thickness and expands its area. The area actuation strain is over $100 \%$. b. Transmittance of the laminate in the visible range as a function of the area actuation strain. Values in $\mathbf{b}$ represent the mean of $n$ tests $(n=3)$.
In summary, here we demonstrated a simple method to reversibly crumple and unfold large-area graphene, which enables us to achieve a set of unprecedented morphologies and properties of graphene, in a controlled manner. A number of future research directions become possible, such as systematic and quantitative investigations of the effects of crumpling on graphene's electrical and electrochemical properties ${ }^{1-3}$ and on the strengths of graphene/polymer interfaces ${ }^{4,5}$. Also, the ridges and vertices in the crumpled graphene are highly deformed and microscopically patterned, which can potentially lead to other new properties and functions, such as patterned chemical reactions ${ }^{31}$ or to applications in biomedical devices. Furthermore, by controlling the microscopic patterns of graphene with a simple macroscopic tool, one can develop new graphene-based systems with novel tunability and flexibility to make nanoscale mechanisms visible at the macroscale.

\section{Methods}

Preparation of crumpled graphene. Few-layer graphene films grown on nickel films on silicon wafers by chemical vapour deposition are purchased from Graphene Supermarket and used as received. A PDMS stamp is adhered to the graphene film on the wafer (Supplementary Fig. S1; ref. 9). The graphene film with the PDMS stamp is detached from the wafer by etching off the nickel film in $1 \mathrm{M} \mathrm{FeCl}_{3}$ solution. The graphene/PDMS sample is rinsed by isopropanol and deionized water and dried in air or nitrogen gas. The cleaned graphene/PDMS sample is stamped on a biaxially pre-stretched (with pre-strain of 200-400\%) elastomer film of VHB acrylic 4905 (3 M, USA) to transfer the graphene film to the elastomer film. Thereafter, the pre-strains in the substrate are relaxed sequentially along two pre-stretched directions. The whole process is schematically illustrated in Step I of Supplementary Fig. S1.

Characterization of microscopic patterns of graphene on elastomer substrates. A scanning electron microscope (SEM, FEI XL30 SEM-FEG) and an atomic force microscope (Digital Instrumentas Dimension 3100) in tapping mode are employed to characterize the morphologies of various patterns on graphene films including wrinkles, delaminated buckles, crumples, and unfolded crumples. 
Measurement of water contact angle. A water drop of $1-3 \mu l$ is placed on the surface of the graphene and images are immediately captured for static contact-angle measurements using a side-view microscope coupled to a camera (Nikon). The water drops are removed by compressed air to dry the graphene surface for repeated contact-angle experiments. The contact angle is measured using the image processing software, ImageJ.

Transmittance measurement. The transmittance of graphene electrodes on elastomer films is measured using an ultraviolet/VIS spectrometer (Cary 6000i, USA) at a wavelength of $550 \mathrm{~nm}$ in the visible range.

Voltage-induced actuation of a graphene-elastomer laminate. Graphene films are transferred to the top and bottom surfaces of a biaxially pre-stretched elastomer film by stamping (Supplementary Fig. S1). The pre-stretches in the elastomer film are relaxed sequentially along two pre-stretched directions to a lower pre-strain. A high-voltage supply (Matsusada, Japan) with controllable ramping rate is used to apply a high voltage between the top and bottom graphene electrodes. The voltage is ramped up to $3,000 \mathrm{~V}$ in $0.05 \mathrm{~S}$ and then reduced to $0 \mathrm{~V}$.

Atomistic simulation of crumpling of graphene. We model the crumpling of a single layer of graphene spanning 100 by $100 \mathrm{~nm}$ (383,125 atoms), confined on a rigid surface. The adaptive intermolecular reactive empirical bond order potential for carbon ${ }^{32}$ is used for full-atomistic molecular dynamics simulations. Van der Waals interactions between the graphene film and the substrate are modelled by a Lennard-Jones 9:3 wall potential corresponding to an adhesive energy of $100 \mathrm{~mJ} \mathrm{~m}^{-2}$ and equilibrium distance of $d=3.35 \AA$. All molecular dynamics simulations are performed using LAMMPS (ref. 33) with a time step of 3 fs. Periodic boundary conditions are applied to the two orthogonal directions parallel to the wall surface. Before loading the graphene film in compression, it is equilibrated for $30 \mathrm{ps}$ in the NVT ensemble using a Langevin thermostat at $300 \mathrm{~K}$. After equilibration, the equibiaxial-compression simulation is performed using the Nose-Hoover thermostat in which the graphene film is scaled down in both $x$ - and $y$-directions by $-0.5 \%$ of the initial length at every 10 ps until the strain reaches $-50 \%$, corresponding to a strain rate of $10^{8} \mathrm{~s}^{-1}$. The sequential-compression simulation is performed with identical conditions but at twice as fast a strain rate along each axis, $210^{8} \mathrm{~s}^{-1}$, to ensure that the total compression time is identical to that of the equibiaxial simulation. The strain rate is chosen such that the observed crumpling pattern has a smaller characteristic scale as the simulation cell size. Because of the finite substrate modulus and thicker graphene film, the overall scale of crumpled morphology cannot be compared directly with experiments, but our simulation results capture the fundamental mechanism and structures of graphene crumpling for the two distinct compression paths.

Received 18 June 2012; accepted 5 December 2012; published online 20 January 2013

\section{References}

1. Miller, J. R., Outlaw, R. A. \& Holloway, B. C. Graphene double-layer capacitor with a.c. line-filtering performance. Science 329, 1637-1639 (2010).

2. Zhu, Y. et al. Carbon-based supercapacitors produced by activation of graphene. Science 332, 1537-1541 (2011).

3. Luo, J. et al. Compression and aggregation-resistant particles of crumpled soft sheets. ACS Nano 5, 8943-8949 (2011).

4. Stankovich, S. et al. Graphene-based composite materials. Nature 442, 282-286 (2006).

5. Ramanathan, T. et al. Functionalized graphene sheets for polymer nanocomposites. Nature Nanotech. 3, 327-331 (2008).

6. Chen, Y. et al. Aerosol synthesis of cargo-filled graphene nanosacks. Nano Lett. 12, 1996-2002 (2012).

7. Pereira, V. M., Castro Neto, A. H., Liang, H. Y. \& Mahadevan, L. Geometry, mechanics, and electronics of singular structures and wrinkles in graphene. Phys. Rev. Lett. 105, 156603 (2010).

8. Geim, A. K. \& Novoselov, K. S. The rise of graphene. Nature Mater. 6, 183-191 (2007).

9. Kim, K. S. et al. Large-scale pattern growth of graphene films for stretchable transparent electrodes. Nature 457, 706-710 (2009).

10. Li, X. et al. Large-area synthesis of high-quality and uniform graphene films on copper foils. Science 324, 1312-1314 (2009).

11. Bao, W. et al. Controlled ripple texturing of suspended graphene and ultrathin graphite membranes. Nature Nanotech. 4, 562-566 (2009).

12. Meyer, J. C. et al. The structure of suspended graphene sheets. Nature 446, 60-63 (2007).

13. Cranford, S. W. \& Buehler, M. J. Packing efficiency and accessible surface area of crumpled graphene. Phys. Rev. B 84, 205451 (2011).
14. Levy, N. et al. Strain-induced pseudo-magnetic fields greater than $300 \mathrm{~T}$ in graphene nanobubbles. Science 329, 544-547 (2010).

15. Guinea, F., Katsnelson, M. I. \& Geim, A. K. Energy gaps and a zero-field quantum Hall effect in graphene by strain engineering. Nature Phys. 6, 30-33 (2010)

16. Scharfenberg, S. et al. Probing the mechanical properties of graphene using a corrugated elastic substrate. Appl. Phys. Lett. 98, 091908 (2011).

17. Li, T. \& Zhang, Z. Substrate-regulated morphology of graphene. J. Phys. D 43, 075303 (2010).

18. Cao, Y. \& Hutchinson, J. W. Wrinkling phenomena in neo-Hookean film/substrate bilayers. J. Appl. Mech. 79, 031019 (2012).

19. Wang, Y. et al. Super-elastic graphene ripples for flexible strain sensors. ACS Nano 5, 3645-3650 (2011).

20. Lee, C., Wei, X., Kysar, J. W. \& Hone, J. Measurement of the elastic properties and intrinsic strength of monolayer graphene. Science 321, 385-388 (2008).

21. Mei, H., Landis, C. M. \& Huang, R. Concomitant wrinkling and buckle-delamination of elastic thin films on compliant substrates. Mech. Mater. 43, 627-642 (2011).

22. Hutchinson, J. W. \& Suo, Z. Mixed-mode cracking in layered materials. Adv. Appl. Mech. 29, 63-191 (1992).

23. Vella, D., Bico, J., Boudaoud, A., Roman, B. \& Reis, P. M. The macroscopic delamination of thin films from elastic substrates. Proc. Natl Acad. Sci. USA 106, 10901-10906 (2009).

24. Rogers, J. A., Someya, T. \& Huang, Y. Materials and mechanics for stretchable electronics. Science 327, 1603-1607 (2010).

25. Kaltenbrunner, M. et al. Ultrathin and lightweight organic solar cells with high flexibility. Nature Commun. 3, 770 (2012).

26. Kim, P., Abkarian, M. \& Stone, H. A. Hierarchical folding of elastic membranes under biaxial compressive stress. Nature Mater. 10, 952-957 (2011).

27. Neinhuis, C. \& Barthlott, W. Characterization and distribution of water-repellent, self-cleaning plant surfaces. Ann. Bot. 79, 667-677 (1997).

28. Rafiee, J. et al. Wetting transparency of graphene. Nature Mater. 11, 217-222 (2012).

29. Zhang, Z., Zhang, T., Zhang, Y. W., Kim, K-S. \& Gao, H. Strain-controlled switching of hierarchically wrinkled surfaces between superhydrophobicity and superhydrophilicity. Langmuir 28, 2753-2760 (2012).

30. Pelrine, R., Kornbluh, R., Pei, Q. B. \& Joseph, J. High-speed electrically actuated elastomers with strain greater than $100 \%$. Science 287, 836-839 (2000).

31. Ruoff, R. A means to an end. Nature 483, S42-S42 (2012).

32. Stuart, S. J., Tutein, A. B. \& Harrison, J. A. A reactive potential for hydrocarbons with intermolecular interactions. J. Chem. Phys. 112, 6472-6486 (2000).

33. Plimpton, S. Fast parallel algorithms for short-range molecular-dynamics. J. Comput. Phys. 117, 1-19 (1995).

\section{Acknowledgements}

The research is primarily funded by the NSF's Research Triangle MRSEC (DMR-1121107), NSF (CMMI-1200515) and NIH (UH2 TR000505). X.Z. acknowledges the support from the Pratt School of Engineering Seed Grant. S.R. and M.J.B. acknowledge the support from AFOSR (FA9550-11-1-0199) and NSF-MRSEC (DMR-0819762). M.J.B. and N.P. acknowledge the support from the MIT-Italy Program (MITOR). N.P. acknowledges the support from the European Research Council under the European Union's Seventh Framework Programme (FP7/2007-2013)/ERC Grant agreement number [279985] (ERC StG Ideas 2011 BIHSNAM). J.Z. and X.Z. acknowledge the help from $\mathrm{C}-\mathrm{H}$. Chen on contact-angle measurements and B. Wiley on transmittance measurements.

\section{Author contributions}

X.Z. conceived the idea, designed and supervised the experiments, and performed the data interpretation. J.Z. designed and carried out the experiments, and performed the data interpretation. Q.W. and Q.T. supported the experiments and contributed to the data interpretation. S.R. and M.J.B. designed, carried out, analysed and interpreted the atomistic simulations. N.P., S.R., M.J.B. and X.Z. developed the theoretical models and interpreted them. X.Z. drafted the manuscript and all authors contributed to the writing of the manuscript.

\section{Additional information}

Supplementary information is available in the online version of the paper. Reprints and permissions information is available online at www.nature.com/reprints. Correspondence and requests for materials should be addressed to X.Z.

\section{Competing financial interests}

The authors declare no competing financial interests. 